\title{
interview
}

\section{Cleaning crystals with light}

\author{
Illumination of a congruent lithium niobate crystal with blue or green light can improve its optical \\ damage threshold dramatically. Nature Photonics spoke to Karsten Buse, who explained that this could \\ result in far cheaper nonlinear crystals for a wide variety of photonic applications.
}

\begin{abstract}
Where did the idea of 'optical cleaning' of lithium niobate come from?

About a decade ago we did intense research on 'thermal fixing', a special method of fixing holograms in photorefractive crystals for applications in holographic data storage. More recently, it occurred to us that the same processes can, if performed correctly, be used to do the opposite: suppress the photorefractive effect in congruent lithium niobate. The photorefractive effect (a light-induced change in refractive index) is problematic because it degrades phase matching in nonlinear crystals, thereby decreasing their efficiency. This led to our work on 'optical cleaning' (reported on page 510 of this issue). It has taken approximately two years to develop the idea and obtain good, reliable data.
\end{abstract}

How does the cleaning scheme work? Light excites electrons from impurities that have energy levels within the band gap of congruent lithium niobate. The bulk photovoltaic effect pushes the electrons in a single, well-defined direction. When heated moderately, the positive ions become mobile and migrate, providing charge compensation. This means that any photoexcitable electrons in the illuminated section of the crystal are 'cleaned' away. A thorough analysis shows that a moving light beam provides the best cleaning effect, as there is a sort of resonance between the speed of the electrons and that of the cleaning light beam - in this situation, the number of remaining electrons decreases exponentially. Simply put, the light works like a brush that sweeps the electrons out of the crystal.

\section{What are the benefits and attractions of this technique?}

Optical cleaning makes congruent lithium niobate useful for nonlinear optical applications. The material is much cheaper than Mg-doped lithium niobate, which is normally used because of its high optical damage threshold. A high-quality Mg-doped periodically poled crystal costs several thousand dollars, but a piece of congruent material costs only a few dollars. However, congruent lithium niobate undergoes photorefractive optical damage at a power



Karsten Buse from the University of Bonn, Germany, is optimistic that his crystal cleaning technique will be useful in industry.

level of only a few milliwatts. Nervertheless, after suitable cleaning, operation at power levels of hundreds-of-milliwatts is possible. Our technique should, in theory, also work with Mg-doped crystals, but this has yet to be confirmed. The scheme could be useful in applications such as high-power whispering gallery resonators. Another attraction is that optical cleaning only requires moderate heating temperatures of $120-180^{\circ} \mathrm{C}$, and can therefore be applied after antireflection coatings have been added to the crystals. The strength of optical cleaning can be tuned by altering the cleaning time.

\section{How can the cleaning performance of the scheme be improved?}

Most of our measurements were performed on iron-doped samples. This allowed us to monitor the cleaning process and hence optimize the cleaning parameters (shape and moving speed of the cleaning light beam, crystal temperature and cleaning time). For applications, however, nominally undoped crystals must be used. We already have a set of parameters that achieves cleaning, but the aim is to fine-tune them to achieve highperformance cleaning in undoped crystals.

How can the scheme be made practical at an industrial scale?

The cleaning process does not require coherent light, allowing cheap blue, green or white light-emitting diodes to be used for the cleaning light. Many cleaning stations can be built for parallel use, which makes it cheap and straightforward to scale the process for large throughput. Even if the overall cleaning takes a few days, this does not delay production significantly because the crystal growth itself already takes a week. From an industrial perspective, the important point is throughput - how many kilograms of material can be cleaned per day, and at what cost. We are very optimistic that by optimizing the cleaning parameters we can improve cleaning speed by an order of magnitude. There is significant interest from industry.

\section{Are there any plans to commercialize the process?}

It is common practice in my research team to file a patent at the beginning of any promising $\mathrm{PhD}$ project. This was also the case for the optical cleaning research, and the patent is now owned by Deutsche Telekom AG. Last year, they gave an exclusive license to a large crystal manufacturer, so it is feasible that this innovation will reach the market.

\section{What are your future plans?}

One of our aims is to optimize the optical cleaning of nominally undoped crystals further. We are also eager to construct nonlinear optical devices that utilize optically cleaned crystals. The next 1-2 years will show how far we can go, and I am sure that publication of this paper will inspire other groups to conduct research in this area. Our current understanding is that there is no fundamental limit to the cleaning; in theory, our treated congruent crystals should be able to reach the damage threshold of Mg-doped crystals. It may also be possible to apply the cleaning scheme to other materials, such as lithium tantalate or perhaps even polymers.

\section{INTERVIEW BY OLIVER GRAYDON}

Karsten Buse and co-workers have a Letter describing their optical cleaning scheme on page 510 of this issue. 